ECCOMAS

\section{Proceedia}

COMPDYN 2021

$8^{\text {th }}$ ECCOMAS Thematic Conference on Computational Methods in Structural Dynamics and Earthquake Engineering

M. Papadrakakis, M. Fragiadakis (eds.)

\title{
INVESTIGATION OF A METHODOLOGY FOR DESCRIBING FAN BLADE FLUTTER LIMITATIONS INDUCED BY NON-LINEAR FRICTION AT BLADE ROOTS
}

\author{
Nicolas Ombret ${ }^{1,2,3}$, Maxime De Pret ${ }^{2}$, Alain Dugeai ${ }^{3}$, Fabrice Thouverez ${ }^{1}$, Laurent \\ Blanc $^{1}$ and Thomas Berthelon ${ }^{3}$ \\ ${ }^{1}$ École Centrale de Lyon, LTDS UMR 5513 \\ 69130 Ecully France \\ e-mail: \{nicolas.ombret,fabrice.thouverez,laurent.blanc\}@ec-lyon.fr \\ ${ }^{2}$ Safran Aircraft Engines \\ 77550 Moissy-Cramayel France \\ e-mail: \{nicolas.ombret,maxime.de-pret\}@safrangroup.com \\ ${ }^{3}$ ONERA - The French Aerospace Lab \\ 92320 Châtillon France \\ e-mail: \{nicolas.ombret-ext,alain.dugeai,thomas.berthelon\}@onera.fr
}

\begin{abstract}
Fan Blade Flutter is an aeroelastic instability which may occur during the operation of a jet engine, depending on the working conditions of the fan stage. It finds its origins in some various mechanisms, including the impact of the environment of the fan stage, which may play an important role in the stability limits due to acoustic effects. If not properly taken into account, flutter can lead to an anticipated ruin of the fan stage as the fluid keeps on giving energy to the structure. However, nonlinear phenomena may appear at some high vibratory amplitude of the blades, resulting in energy dissipation of the aeroelastic system. Blade roots friction is an example of such a case : by dry-friction dissipation at blade roots, a limitation of the vibratory amplitude may be reached, the so-called Limit Cycle Oscillations (LCO), within the unstable regions of the operating domain predicted with a usual linear structural modelling. By taking these nonlinear effects into account, it is then possible to define more precisely the stability limits of the fan stage. In this paper, we describe a methodology to predict LCO induced by blade roots friction, including acoustic effects on stability. First, assuming a linear behaviour of the structure, the stability limits of the fan stage are described using the cyclic symmetry hypothesis and Computational Fluid Dynamics (CFD). Acoustics effects are taken into account by including the fan inlet environment in the numerical model. Then, a nonlinear structural model of the blade is used to compute nonlinear complex modes in order to check for the presence of a LCO. To do so, a reduced model of the fluid response to the blade movement is used. To sum up, this work intends to establish a methodology to be used in an industrial context for the analysis of the nonlinear stability of the fan stage, including LCO phenomena.
\end{abstract}

Keywords: Fan flutter, acoustic reflection, Limit Cycle Oscillation, friction damping, nonlinear complex modes. 


\section{INTRODUCTION}

Aeroelastic stability of fan blades has been studied for decades. It has become more and more important in the recent years to properly predict when this phenomenon does or does not occur, as the reduction of dry mass in jet engines remains one of the toughest objectives for manufacturers to achieve towards less consuming engines to face environmental issues. Moreover, the current trend for manufacturers which consists in developing designs with greater bypass ratio (hence more flexible blades) and shorter inlets allows fluid-structure interactions to have greater effects on blades movement and potentially on its lifecycle. However, physical mechanisms at the origin of fan blade flutter are still not fully understood despite the research done about it and depends on the operating point of the fan stage, as shown in [1].

In this work, we focus on flutter occuring at $75 \%-80 \%$ speed range near stall boundaries. Various experimental and numerical works relate on the mechanisms causing fan blade flutter in this working range, and two major contributions at its origins have been highlighted. The first one refers to a strong interaction between the shock between tip leading edge and mid-chord of the suction side of the blade and the boundary layer around it $[2,3,4,5,6]$. It appears that due to blades vibration and depending on the Inter Blade Phase Angle (IBPA), the shock position may oscillate onto the suction side and be responsible of static pressure fluctuations. In addition, the shock interacts with the boundary layer on the blade surface which may detach behind it. For a periodic motion of the blade, it results in positive (unstable) and negative (stable) aerodynamic work areas onto the blade. This may lead to flutter if the overall aerodynamic work on the blade is positive, meaning that the fluid transfers its energy to the structure. The second mechanism contributing to fan blade flutter in the operating range of interest is due to acoustic interactions between the fan stage and the intake of the engine. Considering a slowly varying intake diameter, acoustic waves may propagate through the intake if the frequency of the perturbation is greater than the caracteristic frequency of acoustic propagation $\omega_{\text {cut-on }}$ called cut-on frequency. Then, if the fan stage blade generates a perturbation at a frequency $\omega$ greater than $\omega_{\text {cut-on }}$, an acoustic wave will propagate through the intake [7]. A reflected wave will occur at the opening of the intake and go back to the fan stage. The phase difference between the wave generated by the fan stage and the one reflected on the intake at the axial position of the stage may cause drastic variation of flutter stability for very narrow operating ranges, yielding the term flutter bite $[8,9,10]$.

Usually, a linear approach of the structure is sufficient to assess the fan stage aeroelastic stability. Two approaches may be used to do so, as well explained in [11] : the first one consists in neglecting the effects of aerodynamics on the fan stage dynamics and then to assess stability by studying aeroelastic stability of the structural modes of the fan with harmonic motion; the second one consists in using strong coupling (partitionned or monolithic approaches) to take into account the effects of the fluid onto the structure dynamics. However, if it is sufficient to consider a linear structure to assess stability limits, it is possible to find stable solutions of the aeroelastic system beyond the stability limits predicted with a linear structure by taking into account nonlinear effects. In this study, we are interested in nonlinear effects due to blade-disk dry friction at blade roots. First works about flutter limitation by dry friction between the blade and the disk seem to be attributed to Sinha and Griffin $[12,13]$. They showed on small degree of freedom systems that in spite of the presence of negative aerodynamic damping, stable and unstable periodic solutions may arise. Since, many works about this subject have been made and new usefull tools have been developped. As we are dealing with nonlinear autonomous systems, there have been many efforts made in order to define the nonlinear dynamics of such systems by 
analogy with the linear modes describing the dynamics of autonomous linear systems. Hence, the notion of nonlinear normal modes has been established, describing the dynamics of conservative autonomous nonlinear systems $[14,15]$ and then extended to nonconservative systems [16]. Other definitions have been suggested in more recent years and are particularly suited to dissipative systems like the one we will study in this paper. Some of these methods are based on frequency approach like the Harmonic Balance Method (HBM) [17, 18]. Decoupled approaches have allowed to find Limit Cycle Oscillations (LCO) of the structure by using the HBM procedure, while modelling aerodynamic effects from Computational Fluid Dynamics (CFD) calculations $[19,20]$. More recently, a fully coupled method in the frequency domain has been established [21] in order to find LCO by using harmonic formulation in both flow and structure models, and iterating in a fixed point loop between the two physics.

In this study, a methodology for predicting LCO induced by dry friction of a fluttering rotor blade will be investigated by the means of nonlinear complex modes [17]. To do so, a decoupled approach will be used. First, the aeroelastic stability of a rotating fan from a typical modern civil engine will be assessed while considering a linear structure. From those results, a simple model of the acoustic effects on flutter stability will be built and used on a phenomenological model representing a blade with nonlinear dry friction effects at its roots. The purpose of this study is to establish a methodology based on nonlinear complex modes that can be applied to the analysis of an industrial fan stage.

\section{AEROELASTIC STABILITY WITHIN THE SCOPE OF A LINEAR STRUCTURE}

\subsection{Case modeling and flow solver}

For this study, a fan model (18 blades) provided by Safran Aircraft Engines has been used with its intake in order to take account of acoustic interactions between these two elements. Figure 1 shows an illustration of this model. Experimental data showed that the fan stage used with this intake became unstable before surge was reached at $75 \%$ speed.



Figure 1: Fan stage model with its intake used for CFD calculations 
The mesh used for this model has to be sufficiently dense in the intake to have a good representation of acoustic waves. However, it is of a higher interest to keep the mesh coarse outside of the intake, where a precise representation of the aerodynamic is not required. As a matter of fact, due to the lack of absorbant acoustic boundary conditions, a too high density grid near the external boundaries would lead to unwanted spurious numerical acoustic reflections, which would not have physical meanings. Hence, a coarsening of the mesh has been put in place from the intake to the outside boundaries of the mesh until eventual acoustic waves coming from the intake are sufficiently dissipated by the spatial scheme. Figure 2 shows the boundaries of the mesh and highlights the ratio between the external characteristic dimension $D$ of the mesh and the intake diameter $d$ midway between the fan and the intake beginning. This ratio has been set to 30. Since there is no Outlet Guide Vanes (OGV) in the model, same considerations have to be taken for both fan primary and secondary outlets. Mesh coarsening has also been used for these parts of the model.
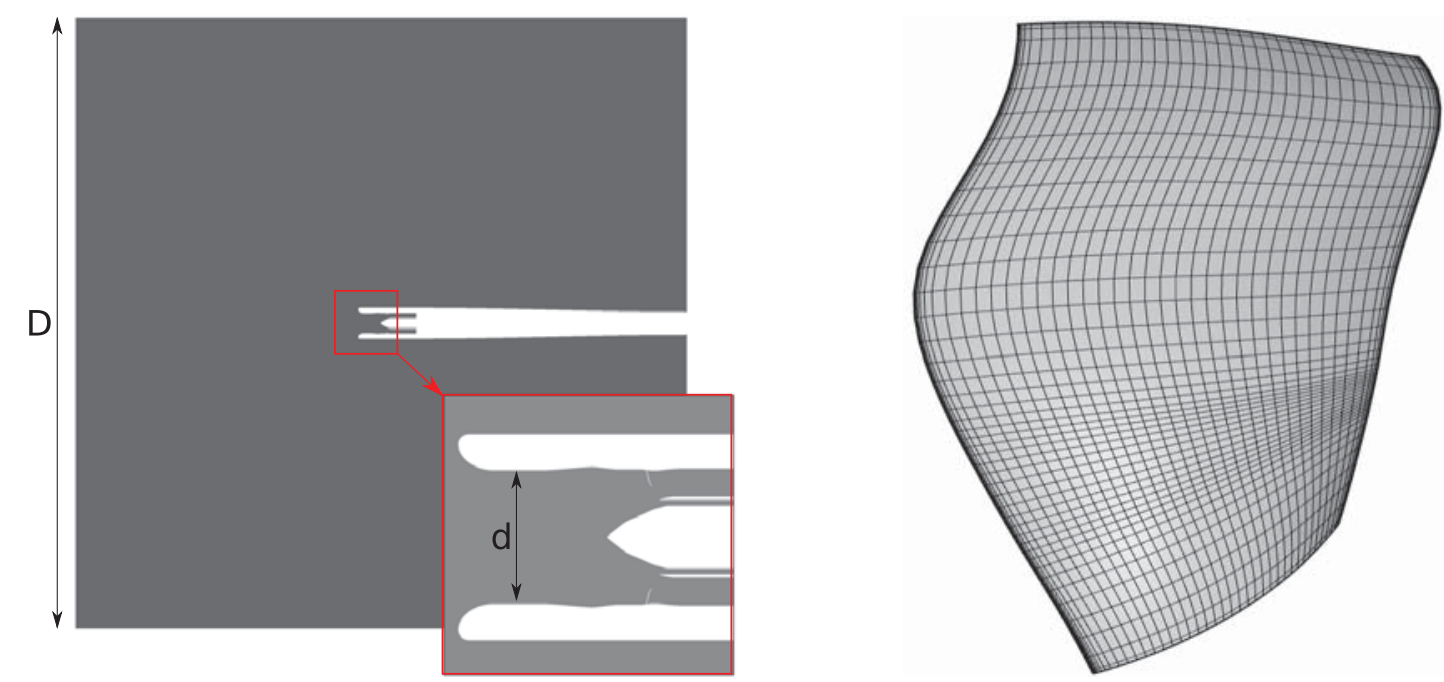

Figure 2: External mesh boundaries on the left, blade mesh on the right

To build the mesh used in this model, the intake and the fan have been meshed separately with two different meshing tools : ICEM for the intake and Autogrid for the fan. Hence, we are dealing with a structured mesh of the full model containing about $11 \mathrm{M}$ cells, with about $3 \mathrm{M}$ cells for the intake and 410000 cells per blade passage.

The solver used to perform both steady and unsteady analysis is the finite-volume elsA software developped at Onera [22, 23], which solves in our case 3D compressible (U)-RANS equations. Roe flux difference splitting scheme has been used, with the two equations turbulence model $k-l$ of Smith. Non reflective boundary conditions have been set upon the external boundaries of the domain with atmospheric sea-level conditions. These conditions are efficient to suppress steady reflections, but are not enough when dealing with unsteady acoustic waves, which justifies the external mesh coarsening. The outflow boundary conditions in primary and secondary outlets is based on radial equilibrium equation for static pressure and allows to select the operating point on the constant $75 \%$ speed of the fan characteristic.

As mentionned earlier, the global mesh is built from two meshes that have been merged : the fan, which rotates at $75 \%$ speed, and the intake which does not move, both in the fixed frame. Therefore, the stator/rotor interface has to be treated to maintain the flow continuity. While performing steady computations, a mixing plane condition is used, transfering azimuthal 
averages of the flow quantities from the intake mesh to the fan one. When dealing with unsteady computations, the mixing plane is replaced with an unsteady interface boundary condition so that acoustic waves may propagate from the fan mesh to the intake mesh. This condition take into account the relative positions of the meshes at the interface at each time step to transfert the information from a cell to its corresponding ones on the other side of the interface. Spatial interpolation is performed when there is no perfect match between the facets of the interface.

\subsection{Aeroelastic model}

As mentioned in the introduction, two approaches may be used to assess the aeroelastic stability of the fan stage. The first one consists in neglecting the impact of the flow on the structure dynamics for the evaluation of aerodynamic forces due to vibrations, while the second one consists in a strong aeroelastic coupling. The approach to be used depends on the case, as the flow may have some impact on the structure dynamics [11]. Even if the second approach may be more precise, it may be computationally expensive to apply, as the first one already gives good insights on the flutter stability while lowering the computationnal costs. In this study, the first approach has been chosen. Strong coupling of the fluid and the structure will be adressed in a future study.

It is assumed for now that the structure is linear and that the vibration is small enough so that aerodynamic forces may be linearized regarding structure movement and velocity. Thus, the aeroelastic stability of the fan may be assessed by considering the aeroelastic stability of each structural eigenmodes separately. Let us consider the equation governing the movement of the linear structure (nonlinear effects due to friction at blade root will be adressed later in this study) :

$$
\mathbf{M} \ddot{\mathbf{x}}+\mathbf{C} \dot{\mathbf{x}}+\mathbf{K x}=\mathbf{f}_{\text {aero }}(\mathbf{x}, \dot{\mathbf{x}})
$$

Solving the eigenvalue problem while considering cyclic symmetry hypothesis for the structure leads to expressing the structure movement in terms of complex modes $\Phi$ representing rotating waves. It is then possible to express the structure movement in the modal basis $\Phi$ :

$$
\mathrm{x}=\Phi \mathrm{q}
$$

By substituing (2) into (1), one can describe the motion of the structure in $\Phi$ with the complex generalized coordinates $q$ [24]. If considering one eigenmode, this leads to the following scalar differential equation :

$$
\mu \ddot{q}+\beta \dot{q}+\gamma q=g_{\text {aero }}(q, \dot{q})
$$

where $\mu, \beta, \gamma$ and $g_{\text {aero }}$ are respectively the generalized mass, generalized dissipation, generalized stiffness and generalized aerodynamic forces.

As we are dealing with stability issues, we consider that the structure is autonomous (i.e there is no external forcing and initial conditions). Moreover, we consider that the movement of the structure is small enough so that the generalized aerodynamic forces may be written as a linear function of the structure movement and velocity (respectively represented by $q$ and $\dot{q}$ ). Hence, (3) may be written as :

$$
\mu \ddot{q}+\beta \dot{q}+\gamma q=A q+B \dot{q}
$$

where :

$$
g_{\text {aero }}(q, \dot{q})=A q+B \dot{q} ; A, B \in \mathbb{C}
$$


Considering harmonic motion $\left(q(t)=q_{0} e^{j \omega_{0} t}\right)$, we have :

$$
\begin{aligned}
g_{\text {aero }} & =\left(A+j \omega_{0} B\right) q \\
& =\left(\left(\operatorname{Re}(A)-\omega_{0} \operatorname{Im}(B)\right)+j \omega_{0}\left(\frac{\operatorname{Im}(A)}{\omega_{0}}+\operatorname{Re}(B)\right)\right) q \\
& =\left(\gamma_{\text {aero }}+j \omega_{0} \beta_{\text {aero }}\right) q
\end{aligned}
$$

In (5), $\gamma_{\text {aero }}$ and $\beta_{\text {aero }}$ may respectively be seen as aerodynamic stiffness and aerodynamic dissipation.

The generalized structural dissipation $\beta$ may be hard to evaluate. Usually, it is convenient to neglect it in order to assess the flutter stability as the approach remains conservative (i.e stability limits will be reached sooner than in a more realistic damped system). Hence, $\beta$ will be omitted in this section. If $\bar{q}$ denotes the conjugate of $q$, the aerodynamic work on one period of harmonic motion may be written as :

$$
\begin{aligned}
W & =\operatorname{Re}\left(\int_{0}^{\frac{2 \pi}{\omega_{0}}} g_{\text {aero }} \overline{\dot{q}} \mathrm{~d} t\right) \\
& =\operatorname{Re}\left(\int_{0}^{\frac{2 \pi}{\omega_{0}}}\left(A+j \omega_{0} B\right) q_{0} e^{j \omega_{0} t}\left(-j \omega_{0} \overline{q_{0}} e^{-j \omega_{0} t}\right) \mathrm{d} t\right) \\
& =2 \pi \omega_{0} \beta_{\text {aero }}\left|q_{0}\right|^{2}
\end{aligned}
$$

From (4), we may express $\beta_{\text {aero }}$ as an aerodynamic damping term $\xi_{\text {aero }}$ :

$$
\xi_{\text {aero }}=-\frac{\beta_{\text {aero }}}{2 \mu \omega_{0}}=-\frac{W}{4 \pi \mu \omega_{0}^{2}\left|q_{0}\right|^{2}}
$$

Flutter occurs when $\xi_{\text {aero }}$ is below zero, meaning that the fluid gives energy to the blade for a blade movement on the considered mode.

\subsection{Stability computations}

Experimental results showed that at $75 \%$ speed, the most unstable mode was the 1F2D for the considered fan stage coupled with its intake. Hence, stability analysis has been performed for this mode. In this conditions, acoustic interactions between the fan and the intake are expected as the eigenfrequency of the mode is greater than the cut-on frequency of some acoustic modes. Figure 3 shows a representation of this mode.

In order to analyse the aeroelastic stability of the selected mode, unsteady aerodynamic simulations with prescribed harmonic motion of the row following its $1 \mathrm{~F} 2 \mathrm{D}$ vibration mode at its eigenfrequency are performed. The simulation are carried out using the Dual Time Stepping (DTS) approach, with 20 dual iterations between each physical timesteps and 192 timesteps per period of vibration. The forward mode is selected (same rotation direction as the one of the fan) to get the largest frequency of perturbation due to vibration in the fixed frame. In these conditions, intake fan acoustic interactions are expected to occur. As a reminder, the perturbation frequency $f$ generated by the fan stage is $f=f_{1 F 2 D}+\Omega \times \mathrm{ND}$ ( $\Omega$ is the rotation frequency of the fan and ND the nodal diameter of the forward mode considered). It has to be greater than the cut-on frequency $f_{c u t-o n}$ so that acoustic waves can propagate through the intake represented as a cylindrical duct. 


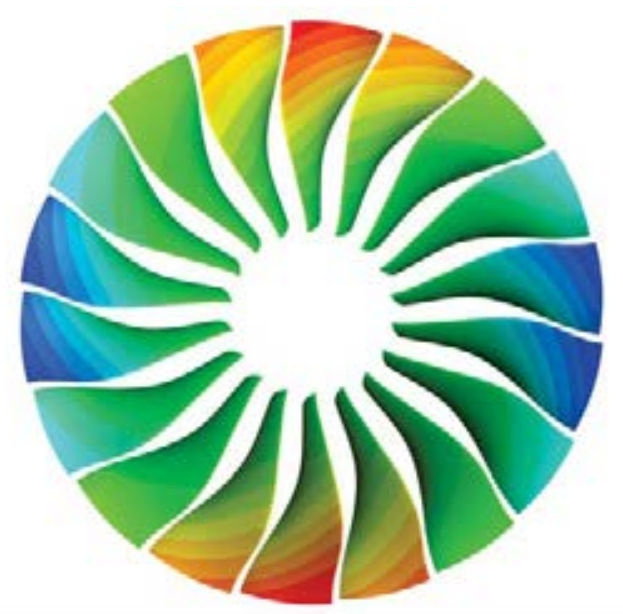

Figure 3: Mode shape for 1F2D

Figure 4 shows the stability analysis results for the 1F2D forward mode, where the evolution of $\xi_{\text {aero }}$ is plotted against the normalized mass flow. The red star represents the stability limits measured during experiments. Stability limit is detected sooner with the numerical model than during experiments, as it was expected since the mechanical damping has been neglected. The approach remains conservative (i.e numerical results do not predict a stable behaviour of the fan on an experimental unstable operating point).

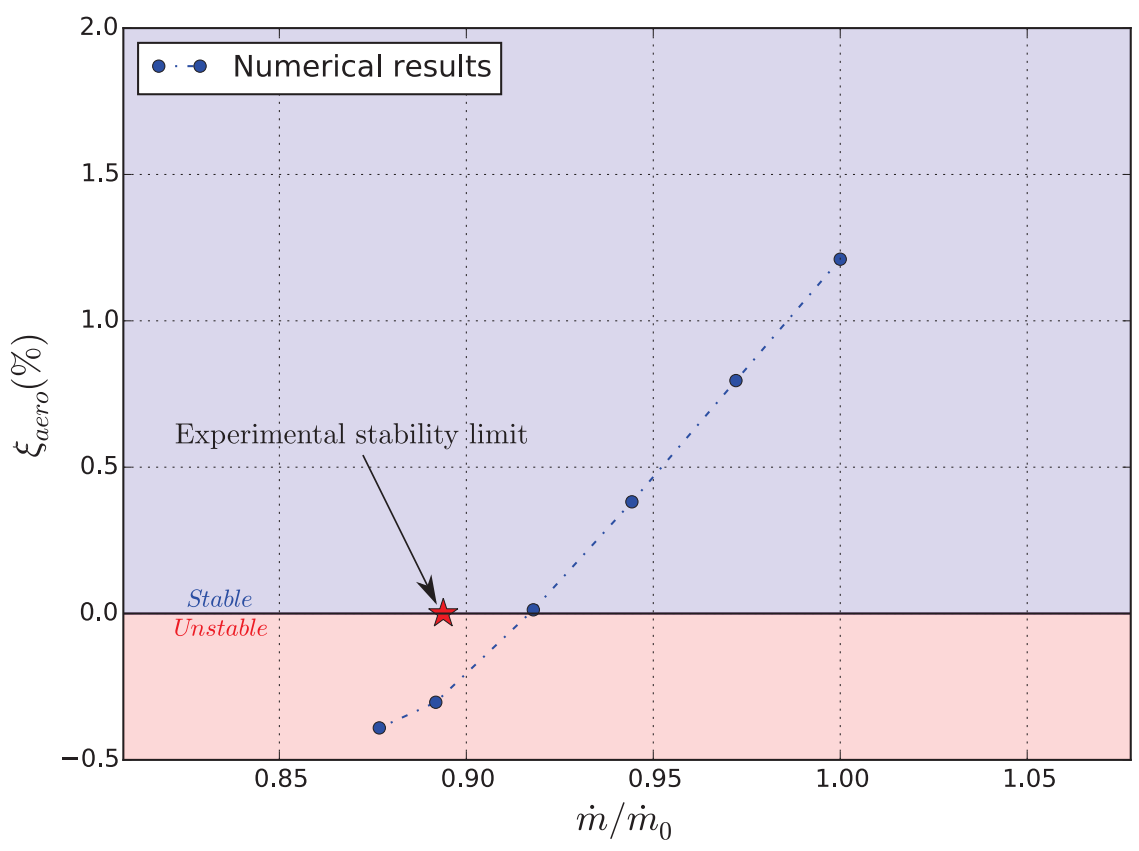

Figure 4: Evolution of $\xi_{\text {aero }}$ on the constant $75 \%$ speed characteristic

For the last period of vibration of the fan, a Discrete Fourier Transform (DFT) has been performed on acoustic variables of interest (density, velocities, static pressure) in the whole domain to check for the presence of acoustic waves in the intake. Results of the DFT of unsteady 
static pressure are displayed in Figure 5. The amplitude representation of static pressure shows a peak in the middle of the intake, which highlights the presence of acoustic interferences between the acoustic waves generated by the vibrating fan and the ones coming from the reflection at the inlet level. The phase reveals a two diameter perturbation in the intake, which was expected as the studied structural mode is a two diameter as well.


Figure 5: Discrete Fourier Transform of the unsteady static pressure in the intake : amplitude on the left, phase on the right

Hence, for mode 1F2D at $75 \%$ speed, acoustic interactions between the fan and the intake may be observed. The impact of this interaction on flutter stability depends on the reflection rate and the phase lag between emitted and reflected acoustic waves, as mentionned in $[9,10]$. Both are functions of the excitation frequency as showed in Bontemps et al. [25]. However, in a context of nonlinear dynamics induced by dry friction, free vibration frequencies of the structure may vary depending on its amplitude of vibration. It is then of interest to have a measure of the sensitivity of $\xi_{\text {aero }}$ against the vibration frequency of the blade to build a model of aerodynamic forces that takes into account acoustic effects on stability.

From the operating point at $\frac{\dot{m}}{\dot{m}_{0}}=0.972$ on the constant $75 \%$ speed characteristic, a set of computations with different values of excitation frequency has been made, whose results are displayed in Figure 6.

It can be seen from this figure that excitation frequency has a first order impact on stability for the considered operating point. A sharp drop in stability appears around $0.87-0.9 f_{1 F 2 D}$, which corresponds to the excitation frequency $f$ equaling the cut-on frequency $f_{\text {cut-on }}$ of the activated acoustic mode in the duct. According to Bontemps et al. [25], this leads to a reflection rate near $100 \%$ and quick variations of the phase lag between emitted and reflected acoustic waves onto the fan blades : in other terms, this is where one may encounter the greatest variation of flutter stability due to acoustic effects and frequency shifts. Results displayed in Figure 6 will be used in the next section as a simplified model of acoustic effects on blade stability. We are now going to introduce the phenomenological nonlinear model used for computing LCO. 




Figure 6: Evolution of $\xi_{\text {aero }}$ against vibration frequency for 1F2D shape mode

\section{NONLINEAR APPROACH FOR FINDING LIMIT CYCLE OSCILLATIONS}

\subsection{Phenomenological model}

The phenomenological model that has been used to represent a blade with dry friction at its root is represented in Figure 7. It is made of three distinct parts : the blade, its root, and the disk. Dry friction nonlinearities are considered between the blade root and the disk. Relative motions authorized between the different parts are rotations. Let us define $X, Y$ and $Z$ the axis of the rotating frame. We assume that there is no rotation of the parts around the $Y$ axis. Hence, we define :

- $\theta_{1, x}$ as the angle of rotation of the disk around $X$;

- $\theta_{1, z}$ as the angle of rotation of the disk around $Z$;

- $\theta_{2, x}$ as the angle between $Z_{\text {root }}$ (axis of the local frame attached to the root) and $Z$;

- $\theta_{2, z}$ as the angle of rotation of the blade root around $Z_{\text {root }}$;

- $\theta_{3, x}$ as the bending angle between $Z_{\text {root }}$ and $Z_{\text {blade }}$ (axis of the local frame attached to the blade) ;

- $\theta_{3, z}$ as the angle of rotation of the blade around $Z_{\text {blade }}$.

Moreover, we consider that the section of the blade containing its center of gravity is at a height $H_{G}$ of its root $\left(H_{G}>>e\right.$ where $e$ is the characteristic dimension of the root thickness). Lastly, we consider that the center of gravity of the blade is not aligned with its center of torsion, but is located at a distance $a$ of it. In that way, when we will consider the modes of the blade with sticking conditions between the blade root and the disk, we will not have pure plunging or twisting modes, but modes with the contributions of those two pure motions.

We are now going to derive the equations of motion of the blade-root-disk system using lagrangian formalism. Some assumptions need to be made first concerning the movement of the 




Figure 7: Phenomenological model of a bending-torsion blade with dry friction at blade root

system. We consider that the disk is motionless in the rotating frame due to its high stiffness (in comparison to those of the root and the blade). Moreover, the root is supposed to rotate only along the $X$ axis due to its high longitudinal dimension in comparison to its thickness. Hence, we have $\theta_{1, x}=\theta_{1, z}=\theta_{2, z}=0$. The system dynamics is then described by the three variables left : $\theta_{2, x}, \theta_{3, x}, \theta_{3, z}$. Let us define the variable $q$ as the vector containing the generalized coordinates of the system $q=\left(\theta_{3, x}, \theta_{3, z}, \theta_{2, x}\right)^{T}$. We consider at last that we have small perturbations (i.e $\theta<<1$ ). For now, the system is assumed to be in vacuum. The Lagrange equations are :

$$
\frac{\mathrm{d}}{\mathrm{d} t} \frac{\partial \mathcal{L}}{\partial \dot{q}}-\frac{\partial \mathcal{L}}{\partial q}=\mathcal{Q}
$$

where $\mathcal{Q}$ is the generalized non conservative torque (here, due to blade root friction), and :

$$
\mathcal{L}=\mathcal{T}-\mathcal{V}
$$

In these equations, $\mathcal{T}$ is the kinetic energy, while $\mathcal{V}$ is the potential energy of the system. Let us denote respectively (1), (2) and (3) the disk, the root and the blade. Then, we have :

$$
\mathcal{T}=\mathcal{T}_{2}+\mathcal{T}_{3}
$$

with :

$$
\begin{aligned}
\mathcal{T}_{2} & =\frac{1}{2} J_{2 x} \dot{\theta}_{2 x}^{2} \\
\mathcal{T}_{3} & =\frac{1}{2}\left(m_{3} v_{G}^{2}+\Omega_{3} \cdot\left(\overline{\bar{I}}_{G, 3} \cdot \Omega_{3}\right)\right)
\end{aligned}
$$

$J_{2 x}, m_{3}, v_{G}, \Omega_{3}$ and $\overline{\bar{I}}_{G, 3}$ are respectively the moment of inertia of the blade root around $X$, the mass of the blade, the velocity of the center of gravity of the blade in the laboratory frame 
of reference, the angular velocity vector and the moment of inertia tensor of the blade expressed at its center of mass $G$.

By calculation, we have at first order :

$$
v_{G}^{2}=a^{2} \dot{\theta}_{3 z}^{2}-2 a H_{G} \dot{\theta}_{3 z}\left(\dot{\theta}_{2 x}+\dot{\theta}_{3 x}\right)+H_{G}^{2}\left(\dot{\theta}_{2 x}+\dot{\theta}_{3 x}\right)^{2}+a^{2}\left(\dot{\theta}_{2 x}+\dot{\theta}_{3 x}\right)^{2} \theta_{3 z}^{2}
$$

Since small perturbations are considered $(\theta<<1)$, it seems reasonable to neglect the last term of this equation, which is of higher order than the other terms :

$$
v_{G}^{2}=a^{2} \dot{\theta}_{3 z}^{2}-2 a H_{G} \dot{\theta}_{3 z}\left(\dot{\theta}_{2 x}+\dot{\theta}_{3 x}\right)+H_{G}^{2}\left(\dot{\theta}_{2 x}+\dot{\theta}_{3 x}\right)^{2}
$$

Under the assumption that the blade has symmetry properties around its center of torsion on the section containing the center of mass, the moment of inertia tensor of the blade is written as follow :

$$
\overline{\bar{I}}_{G, 3}=\left(\begin{array}{ccc}
J_{2 x} & 0 & 0 \\
0 & J_{3 y} & 0 \\
0 & 0 & J_{3 z}
\end{array}\right)
$$

The angular velocity vector of the blade is written :

$$
\Omega_{3}=\left(\begin{array}{c}
\dot{\theta}_{2 x}+\dot{\theta}_{3 x} \\
0 \\
\dot{\theta}_{3 z}
\end{array}\right)
$$

Hence, we have :

$$
\begin{aligned}
\mathcal{T}=\frac{1}{2} J_{2 x} \dot{\theta}_{2 x}^{2} & +\frac{1}{2} m_{3} a^{2} \dot{\theta}_{3 z}^{2}-m_{3} a H_{G} \dot{\theta}_{3 z}\left(\dot{\theta}_{2 x}+\dot{\theta}_{3 x}\right)^{2}+\frac{1}{2} m_{3} H_{G}^{2}\left(\dot{\theta}_{2 x}+\dot{\theta}_{3 x}\right)^{2} \\
& +\frac{1}{2} J_{3 x}\left(\dot{\theta}_{2 x}+\dot{\theta}_{3 x}\right)^{2}+\frac{1}{2} J_{3 z} \dot{\theta}_{3 z}^{2}
\end{aligned}
$$

For the potentiel energy of the system, some rotational stiffnesses are considered between each part. Hence, it may be written as follow :

$$
\mathcal{V}=\frac{1}{2} C_{23 z} \theta_{3 z}^{2}+\frac{1}{2} C_{23 x} \theta_{3 x}^{2}+\frac{1}{2} C_{12 x} \theta_{2 x}^{2}
$$

Using equations (14) and (15) in (8) leads to the following three degree of freedom system :

$$
I\left(\begin{array}{c}
\ddot{\theta}_{3 x} \\
\ddot{\theta}_{3 z} \\
\ddot{\theta}_{2 x}
\end{array}\right)+C\left(\begin{array}{c}
\theta_{3 x} \\
\theta_{3 z} \\
\theta_{2 x}
\end{array}\right)=\mathcal{Q}
$$

with :

$$
I=\left(\begin{array}{ccc}
m_{3} H_{G}{ }^{2}+J_{3 x} & -a m_{3} H_{G} & m_{3} H_{G}^{2}+J_{3 x} \\
-a m_{3} H_{G} & m_{3} a^{2}+J_{3 z} & -a m_{3} H_{G} \\
m_{3} H_{G}{ }^{2}+J_{3 x} & -a m_{3} H_{G} & m_{3} H_{G}{ }^{2}+J_{2 x}+J_{3 x}
\end{array}\right)
$$


and :

$$
C=\left(\begin{array}{ccc}
C_{23 x} & 0 & 0 \\
0 & C_{23 z} & 0 \\
0 & 0 & C_{12 x}
\end{array}\right)
$$

$\mathcal{Q}$ is a nonlinear torque due to the presence of a friction force between the blade root and the disk. In this approach, the nonlinear friction torque is simply modelled using the following regularized form of the Coulomb law, which will be helpfull to derive the jacobian of the nonlinear algebraic system that will be solved in the next subsection :

$$
\mathcal{Q}=\left(\begin{array}{c}
0 \\
0 \\
-e \mu N \tanh \left(p \frac{\dot{\theta}_{2 x}}{e \mu N}\right)
\end{array}\right)
$$

The evolution of the nonlinear torque is plotted in Figure 8. As it can be seen, a $p$ parameter allows to choose the slope of the nonlinearity : the nonlinear force asymptotically approaches the Coulomb law as one chooses a high value for $p$.

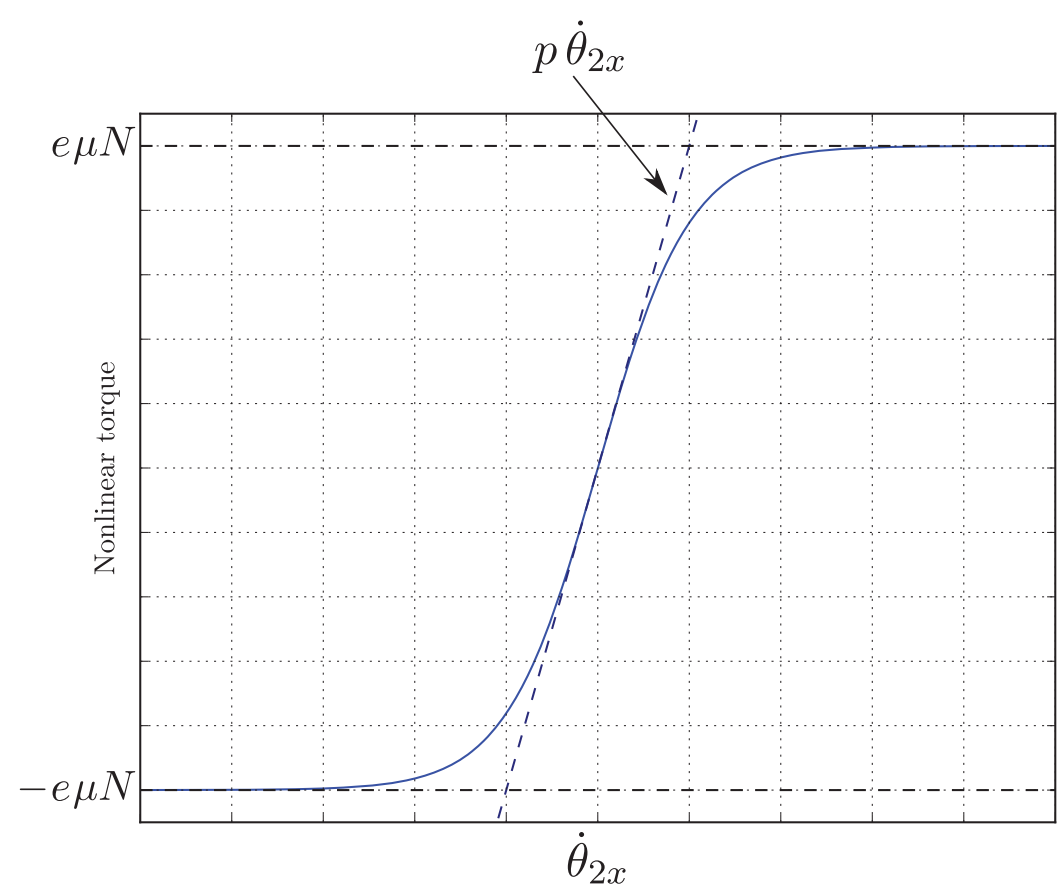

Figure 8: Evolution of the nonlinear torque against the movement of the blade root

Now that the equations of the nonlinear dynamics of the system have been written, we are going to use nonlinear complex modes in order to analyse the occurence of LCO for the bladeroot-disk system.

\subsection{Nonlinear complex modes}

The notion of nonlinear complex mode [17] has been introduced to characterize the nonlinear dynamics of nonconservative autonomous systems. Its formulation is built by analogy with 
linear complex modes, as the eigenvalues of such a system may be written in the form :

$$
\lambda=\beta+j \omega
$$

Let us take the general equation of movement of a structure in presence of some dry friction nonlinear force :

$$
\mathbf{M} \ddot{\mathbf{x}}+\mathbf{C} \dot{\mathbf{x}}+\mathbf{K} \mathbf{x}+\mathbf{f}_{\mathbf{n l}}(\mathbf{x}, \dot{\mathbf{x}})=0
$$

We suppose the solution of this differential equation being in the following nonlinear complex mode form :

$$
\mathbf{x}(t)=\mathbf{a}_{0}+\sum_{k=1}^{N_{h}} e^{-k \beta t}\left(\mathbf{a}_{k} \cos (k \omega t)+\mathbf{b}_{k} \sin (k \omega t)\right)
$$

Two time scales may be seen in equation (21): the first time scale is due to the $\beta$ term, which may be defined as the dissipation of the nonlinear complex mode by analogy with linear complex mode ; the second time scale is due to the $\omega$ term. We suppose that the time scale associated to $\omega$ is much faster than the one associated to $\beta$ (i.e $\omega>>\beta$ ). Moreover, $\beta$ is supposed to be the same for each harmonic. Defined in that way, the main difference between nonlinear complex modes and linear complex modes is the presence of multi-harmonic content in the nonlinear case.

By the means of a Galerkine procedure, one may rewrite the nonlinear differential equation of the structure into an algebraic one. To do so, a scalar product must be chosen. The scalar product that we consider is the inner product of square integrable functions defined as :

$$
\forall f, g \in \mathcal{C}\left(0 ; \frac{2 \pi}{\omega}\right)^{2},\langle f \mid g\rangle=\frac{\omega}{\pi} \int_{0}^{\frac{2 \pi}{\omega}} f(t) \overline{g(t)} d t
$$

Let us consider the Fourier basis functions $1, \cos (k \omega t), \sin (k \omega t) \forall k \in\left\{1 ; N_{h}\right\}$. By reinjecting (21) into the equations of motion and applying the inner product (22) with the Fourier basis functions, the dynamics of the nonlinear system may be described by the following algebraic equation in residual form :

$$
\mathcal{R}(\mathbf{X}, \omega, \beta)=\mathbf{Z}(\omega, \beta) \mathbf{X}+\mathbf{F}_{\mathbf{n l}}(\mathbf{X}, \omega, \beta)=0
$$

where the multi-harmonic stiffness matrix $\mathbf{Z}$ is :

$$
\mathbf{Z}=\left(\begin{array}{cccccc}
2 \mathbf{K} & & & & & \\
& \mathbf{Z}^{1} & & & & \\
& & \ddots & & & \\
& & & \mathbf{Z}^{k} & & \\
& & & & \ddots & \\
& & & & & \mathbf{Z}^{N_{h}}
\end{array}\right)
$$

and :

$$
\mathbf{Z}^{k}=\left(\begin{array}{cc}
\mathbf{K}+\left((k \beta)^{2}-(k \omega)^{2}\right) \mathbf{M}-k \beta \mathbf{C} & k \omega \mathbf{C}-2 k^{2} \beta \omega \mathbf{M} \\
-\left(k \omega \mathbf{C}-2 k^{2} \beta \omega \mathbf{M}\right) & \mathbf{K}+\left((k \beta)^{2}-(k \omega)^{2}\right) \mathbf{M}-k \beta \mathbf{C}
\end{array}\right)
$$


To perform the scalar product and derive (23), the hypothesis $\omega>>\beta$ has been used to neglect the amplitude variation of $\mathbf{x}$ due to the dissipation $\beta$ during a period $\frac{2 \pi}{\omega}$. Hence, orthogonality properties may be used to derive the scalar product of the equation of movement with the Fourier basis functions.

In (23), the unknowns are $\mathbf{X}, \omega$ and $\beta$. As the system is autonomous, there are two more unknowns in the system than there are equations, which means that we are dealing with a rectangular system. By adding two more equations, it is possible to make it square. The first equation may be seen as a constraint equation, while the second one as a phase equation. By considering arc-length continuation [26] and a zero phase for an arbitrary harmonic $k$ and degree of freedom $q$, these two equations may be written as :

$$
\left\{\begin{array}{l}
\mathcal{R}_{\text {arc }}(\mathbf{X}, \omega, \beta)=\left\|\mathbf{X}-\mathbf{X}_{0}\right\|^{2}+\left\|\omega-\omega_{0}\right\|^{2}+\left\|\beta-\beta_{0}\right\|^{2}-\Delta s^{2}=0 \\
\mathcal{R}_{\text {phase }}(\mathbf{X}, \omega, \beta)=\mathbf{b}_{k, q}=0
\end{array}\right.
$$

where $\Delta s$ is the arc-lentgh radius.

A Newton-Raphson procedure is used to solve (23) and (26) simultaneously. Thus, an evaluation of the nonlinear forces in the frequency domain has to be done. Since there is no direct analytical expression of $\mathbf{F}_{\mathbf{n l}}(\mathbf{X}, \omega, \beta)$, an Alternating Frequency-Time (AFT) method is used, as described in [27]. As previously, under the hypothesis that two different time scales are considered, a periodic movement of the structure is assumed during a pseudo-period $\frac{2 \pi}{\omega}$, which means that the decrease due to $\beta$ when performing the AFT method is neglected [17]. It is then assumed that the decrease of the nonlinear forces is the same than the one of the movement.

To get a full branch of nonlinear complex mode, the computation needs to be initialized. To do so, the system is set with small amplitudes of vibration so that the linear sticking case is a good approximation of the solution. Then, the first solution is searched in a subspace of the solutions of (23) at a distance $\Delta s$ from the linear sticking case with the Newton-Raphson procedure. When converged, the computed solution is stored on one hand and replaces $\left(\mathbf{X}_{0}, \omega_{0}, \beta_{0}\right)$ on the other hand. The next solution is predicted using secant predictor [28]. It is then computed with the Newton-Raphson procedure and so on.

\subsection{Fluid modeling}

We are here interested in taking into account aerodynamic effects on the nonlinear complex mode computation. First, let us consider the asymptotic case when there are sticking conditions between the blade root and the disk (i.e $\theta_{2, x}=0$ ). Under the hypothesis that the modal basis of the full blade model used in elsA and the phenomenological model are equivalent on the considered bending mode, we assume that we may transfer the aerodynamic damping computed in aeroelastic computations to the equivalent bending mode of the phenomenological model. From (16), we write the movement equation in sticking conditions where $\theta_{2, x}=0$ :

$$
I_{\text {stick }}\left(\begin{array}{c}
\ddot{\theta}_{3 x} \\
\ddot{\theta}_{3 z}
\end{array}\right)+C_{\text {stick }}\left(\begin{array}{c}
\theta_{3 x} \\
\theta_{3 z}
\end{array}\right)=0
$$

with :

$$
I_{\text {stick }}=\left(\begin{array}{cc}
m_{3} H_{G}{ }^{2}+J_{3 x} & -a m_{3} H_{G} \\
-a m_{3} H_{G} & m_{3} a^{2}+J_{3 z}
\end{array}\right)
$$

and :

$$
C_{\text {stick }}=\left(\begin{array}{cc}
C_{23 x} & 0 \\
0 & C_{23 z}
\end{array}\right)
$$


Solving the eigenvalue problem associated to (27) allows to find the modal basis $\Phi$ of the system in sticking conditions. It is possible to switch from physical base to modal basis using :

$$
\left(\begin{array}{c}
\theta_{3 x} \\
\theta_{3 z}
\end{array}\right)=\Phi\left(\begin{array}{c}
q_{\text {bend }} \\
q_{\text {torsion }}
\end{array}\right)
$$

When switching equation (27) to modal basis in sticking conditions, we have :

$$
\mu\left(\begin{array}{c}
\ddot{q}_{\text {bend }} \\
\ddot{q}_{\text {torsion }}
\end{array}\right)+\gamma\left(\begin{array}{c}
q_{\text {bend }} \\
q_{\text {torsion }}
\end{array}\right)=0
$$

with :

$$
\mu=\Phi^{T} I_{\text {stick }} \Phi=\left(\begin{array}{cc}
\mu_{\text {bend }} & 0 \\
0 & \mu_{\text {torsion }}
\end{array}\right)
$$

and :

$$
\gamma=\Phi^{T} C_{\text {stick }} \Phi=\left(\begin{array}{cc}
\gamma_{\text {bend }} & 0 \\
0 & \gamma_{\text {torsion }}
\end{array}\right)
$$

Under the assumption that we may use the aerodynamic damping previously computed, a damping modal matrix is added to (31) in the form :

$$
\mu\left(\begin{array}{c}
\ddot{q}_{\text {bend }} \\
\ddot{q}_{\text {torsion }}
\end{array}\right)+\left(\begin{array}{cc}
2 \sqrt{\mu_{\text {bend }} \gamma_{\text {bend }}} \xi_{\text {aero }} & 0 \\
0 & 0
\end{array}\right)\left(\begin{array}{c}
\dot{q}_{\text {bend }} \\
\dot{q}_{\text {torsion }}
\end{array}\right)+\gamma\left(\begin{array}{c}
q_{\text {bend }} \\
q_{\text {torsion }}
\end{array}\right)=0
$$

Going back in the physical basis gives :

$$
I_{\text {stick }}\left(\begin{array}{c}
\ddot{\theta}_{3 x} \\
\ddot{\theta}_{3 z}
\end{array}\right)+D_{\text {stick }}\left(\begin{array}{c}
\dot{\theta}_{3 x} \\
\dot{\theta}_{3 z}
\end{array}\right)+C_{\text {stick }}\left(\begin{array}{c}
\theta_{3 x} \\
\theta_{3 z}
\end{array}\right)=0
$$

with :

$$
D_{\text {stick }}=\Phi^{-T}\left(\begin{array}{cc}
2 \sqrt{\mu_{\text {bend }} \gamma_{\text {bend }}} \xi_{\text {aero }} & 0 \\
0 & 0
\end{array}\right) \Phi^{-1}
$$

where $\Phi$ is the eigenvectors matrix of the system with sticking conditions. Since aerodynamic efforts are applied only on the blade, we make the assumption that $\theta_{2 x}$ will not be affected by aerodynamic damping. Hence, we may use the aerodynamic dissipation matrix $D_{\text {stick }}$ in the full model as :

$$
I\left(\begin{array}{c}
\ddot{\theta}_{3 x} \\
\ddot{\theta}_{3 z} \\
\ddot{\theta}_{2 x}
\end{array}\right)+D\left(\begin{array}{c}
\dot{\theta}_{3 x} \\
\dot{\theta}_{3 z} \\
\dot{\theta}_{2 x}
\end{array}\right)+C\left(\begin{array}{c}
\theta_{3 x} \\
\theta_{3 z} \\
\theta_{2 x}
\end{array}\right)=\mathcal{Q}
$$

with :

$$
D=\left(\begin{array}{ccc}
D_{\text {stick }} & 0 \\
0 & 0 & 0
\end{array}\right)
$$


Nonlinear complex modes may now be computed as in the previous subsection from (37) by taking into account fluid effects. It is at last assumed that the aerodynamic damping only affects the first harmonic of the nonlinear complex mode : as higher-harmonic content is expected to be much lower than the fundamental content, we may suppose that it does not affect aerodynamic forces. The multi-harmonic stiffness matrix can be expressed as follow :

$$
\mathbf{Z}=\left(\begin{array}{cccccc}
2 C & & & & & \\
& \mathbf{Z}^{1} & & & & \\
& & \ddots & & & \\
& & & \mathbf{Z}^{k} & & \\
& & & & \ddots & \\
& & & & & \mathbf{Z}^{N_{h}}
\end{array}\right)
$$

with :

$$
\mathbf{Z}^{1}=\left(\begin{array}{cc}
C+\left(\beta^{2}-\omega^{2}\right) I-\beta D & \omega D-2 \beta \omega I \\
-(\omega D-2 \beta \omega I) & C+\left(\beta^{2}-\omega^{2}\right) I-\beta D
\end{array}\right)
$$

and $\forall k \neq 1$ :

$$
\mathbf{Z}^{k}=\left(\begin{array}{cc}
C+\left((k \beta)^{2}-(k \omega)^{2}\right) I & -2 k^{2} \beta \omega I \\
2 k^{2} \beta \omega I & C+\left((k \beta)^{2}-(k \omega)^{2}\right) I
\end{array}\right)
$$

In (40), note that the aerodynamic dissipation matrix $D$ depends on the free frequency $\omega$, meaning that acoustic effects are taken into account.

\section{NUMERICAL RESULTS}

\subsection{Model identification process}

The model derived in the previous section has been used to compute nonlinear complex modes. It should be noticed that in the formulation of $I$, all of the terms may be extracted from any FEM model of blade. In this case, the values have been extracted from the blade model used previously in the aeroelastic computations. The remaining terms to choose are those in $C$. To do so, let us remind that the nonlinear behaviour of the system is bounded by two asymptotical linear cases respectively defined by sticking or sliding conditions between the blade root and the disk. Hence, rotational stiffnesses have been set so that the eigenfrequencies of the phenomenological blade-root-disk system on asymptotical cases match the eigenfrequencies of the model used for aeroelastic computations on the same asymptotical cases. Table 1 sums up the values that have been used for the phenomenological model. Eigenfrequencies and eigenvectors of the asymptotical linear cases are reported in Table 2.

\subsection{Nonlinear analysis in vacuum}

Results for a branch of nonlinear complex mode are displayed in Figures 9 and 10. The evolutions of free frequency and modal nonlinear damping expressed as $\frac{\beta}{\omega}$ against the amplitude of the first harmonic of $\theta_{3 x}$ are plotted in the first one. Trajectories in the configuration space of the dynamics of the system during a pseudo-period are plotted in the second one. The trajectories of Figure 10 match the dots of Figure 9. 


\begin{tabular}{cc}
\hline \hline Parameter & Value \\
\hline$m_{3}$ & 4.3 \\
$H_{G}$ & 0.27 \\
$a$ & -chord $\times 0.15$ \\
$J_{3 x}\left(\times 10^{-6}\right)$ & 159114 \\
$J_{3 z}\left(\times 10^{-6}\right)$ & 28120 \\
$J_{2 x}\left(\times 10^{-6}\right)$ & 217 \\
$C_{23 x}$ & 119500 \\
$C_{23 z}$ & 40000 \\
$C_{12 x}$ & 110000 \\
$e$ & 0.02 \\
$\mu$ & 0.1 \\
$N$ & 200000 \\
$p$ & 123078 \\
$N_{h}$ & 15 \\
\hline \hline
\end{tabular}

Table 1: Numerical values of the phenomenological model

\begin{tabular}{ccccc}
\hline \hline Asympt. case & $f$ & $\theta_{3 x}$ & $\theta_{3 z}$ & $\theta_{2 x}$ \\
\hline Sticking & $f_{1 F 2 D}$ & -0.919 & -0.395 & 0. \\
Sliding & $0.7 \times f_{1 F 2 D}$ & 0.655 & 0.252 & 0.712 \\
\hline \hline
\end{tabular}

Table 2: Eigenfrequencies and eigenvectors of the asymptotical linear cases


Figure 9: Nonlinear complex mode of the phenomenological model in vacuum 

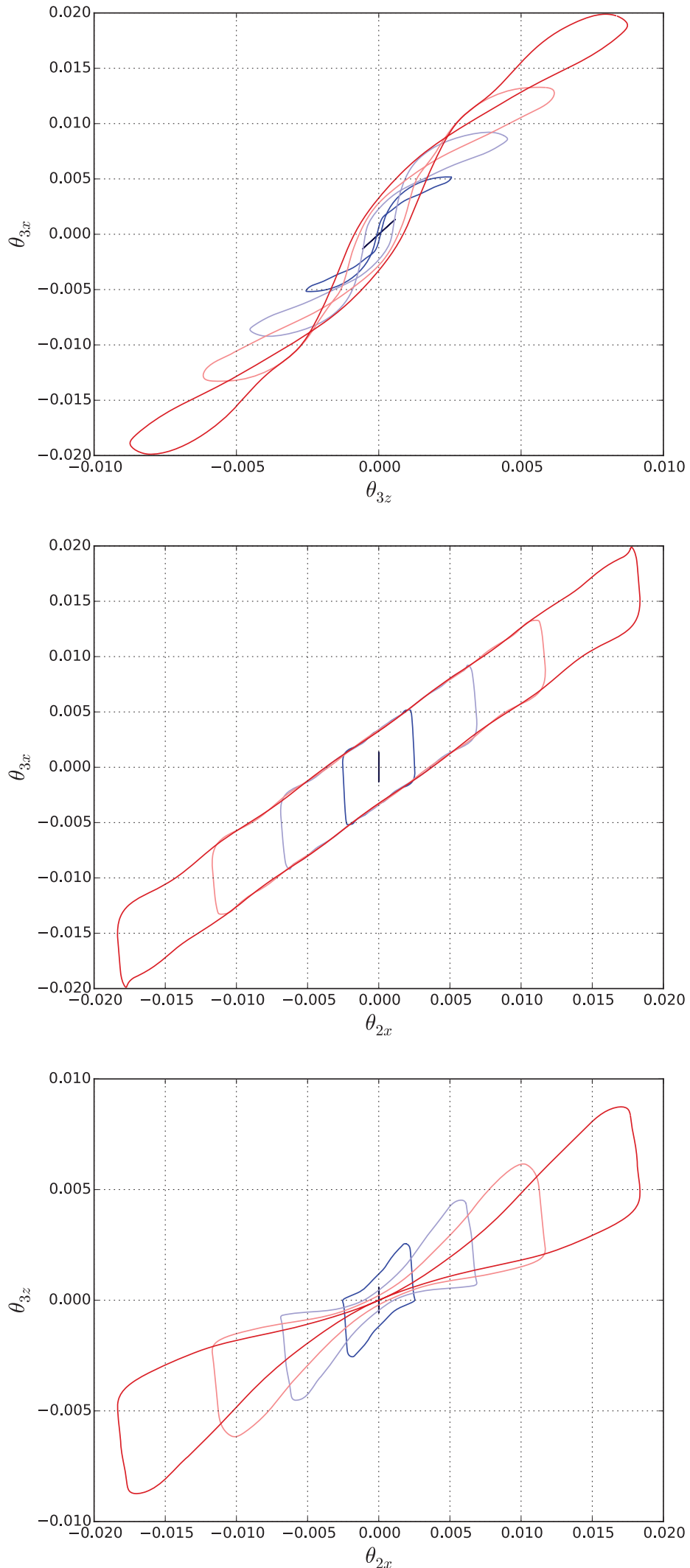

Figure 10: Nonlinear dynamics of the system in the configuration space 
The nonlinear behaviour of the system dynamics changes as expected with increasing levels of $\theta_{3 x, 1}$ (i.e increasing levels of vibration energy). A frequency shift is observed in Figure 9, from the frequency of the linear sticking case to the frequency of the linear sliding one. This frequency shift was expected, as blade root friction may be seen as the liberation of a degree of freedom which reduces the stiffness of the system, thus the free frequency. As $\theta_{3 x, 1}$ increases, one may see when nonlinearities start to have a significant impact on the system dynamics, as sharp changes may be observed on the nonlinear damping and free frequency.

It can be seen in Figure 10 the effects of friction nonlinearities onto the system dynamics. Starting in sticking conditions (deep blue curves), the system dynamics evolves along a straight line as the system is nearly linear. With increasing levels of friction nonlinearities (from deep blue to deep red), one may observe an opening and a curvature of the generalized coordinates trajectories. A phase difference between the generalized coordinates appears, leading to hysteresis cycles of the system dynamics. Such cycles are typical of systems with dissipation due to friction nonlinearities.

The system dynamics has been caracterized with nonlinear complex modes in vacuum. The previously derived simplified fluid representation is going to be added to the system, using numerical results of the first section.

\subsection{Nonlinear analysis with simplified fluid representation}

In this study, we are interested in predicting LCO with nonlinear complex modes while taking into account flutter instability and changes in acoustic effects due to frequency shifts. To do so, the methodology derived in subsection 3.3 and the results exposed in Figure 6 will be used. A spline has been created to caracterize the evolution of $\xi_{\text {aero }}$ with respect to the frequency of vibration. In that way, the evolution of $\xi_{\text {aero }}$ is in regards to $f$, and the jacobian may be analytically derived easily. Figure 11 illustrates the spline used for computations.

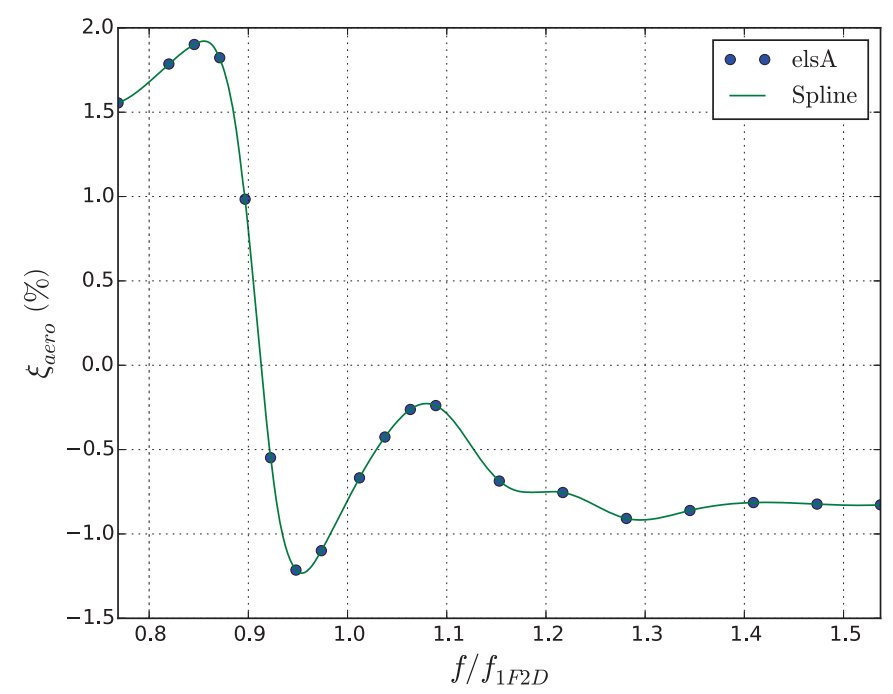

Figure 11: $\xi_{\text {aero }}$ spline implemented in the computation of the nonlinear complex mode

It should be noticed that Figure 11 differs from $6:$ the points have been shifted to a lower level in the latter so that we are in presence of negative aerodynamic damping when the system is stuck and vibrates at $f=f_{1 F 2 D}$. This shift, which is artificial, has been applied so that the 
system may be unstable in absence of friction nonlinearities. The methodology can then be tested to assess the presence of LCO when taking into account aerodynamic effects. Another way to proceed would have been to compute the dependance of $\xi_{\text {aero }}$ against $\omega$ on a operating point beyond the stability limit computed previously. It is assumed that it would have produced similar results. A nonlinear complex mode has been computed when the system is initialized on the unstable bending mode. Numerical parameters have been kept the same as the case in vacuum. Results are displayed in Figure 12.
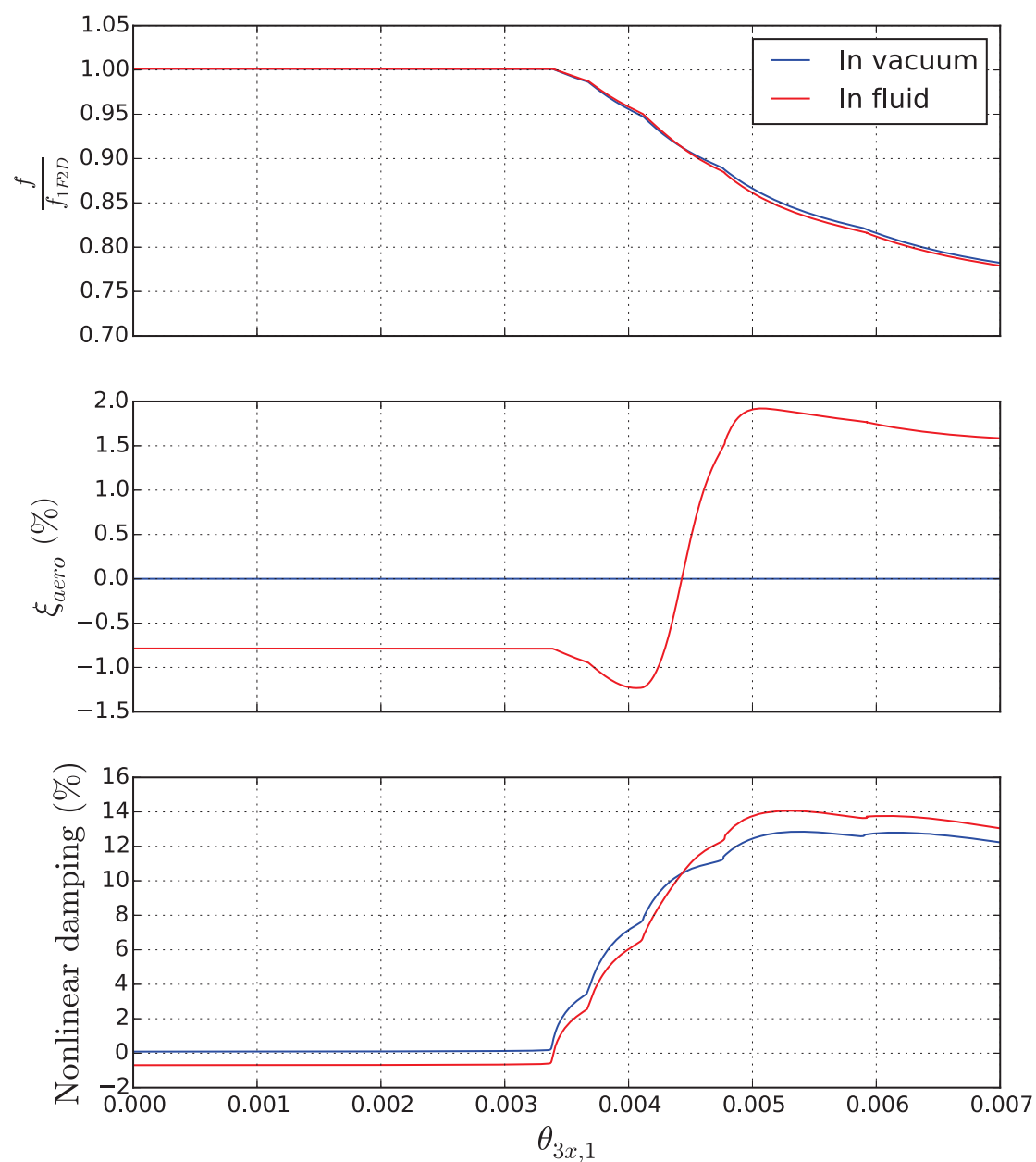

Figure 12: Nonlinear complex mode of the phenomenological model with fluid modeling

Since there is no more information on $\xi_{\text {aero }}$ for values of $f$ under $0.77 f_{1 F 2 D}$, the computation has been interrupted for this value of $f$. It can be seen that qualitatively we have the same trends for $f$ and the nonlinear damping as in the case in vacuum, in spite of small discrepancies. What must be noticed is that there is a point on the nonlinear complex mode where the nonlinear damping equals zero, which highlights the presence of a LCO on this point. By using a direct Fourier transform, it is possible to display the dynamics of the system in the state space on the LCO, as shown in figure 13. Finally, it may be seen in Figure 12 that the evolution $\xi_{\text {aero }}$ follows the evolution of the free frequency with increasing values of amplitude, as implemented from Figure 11. 

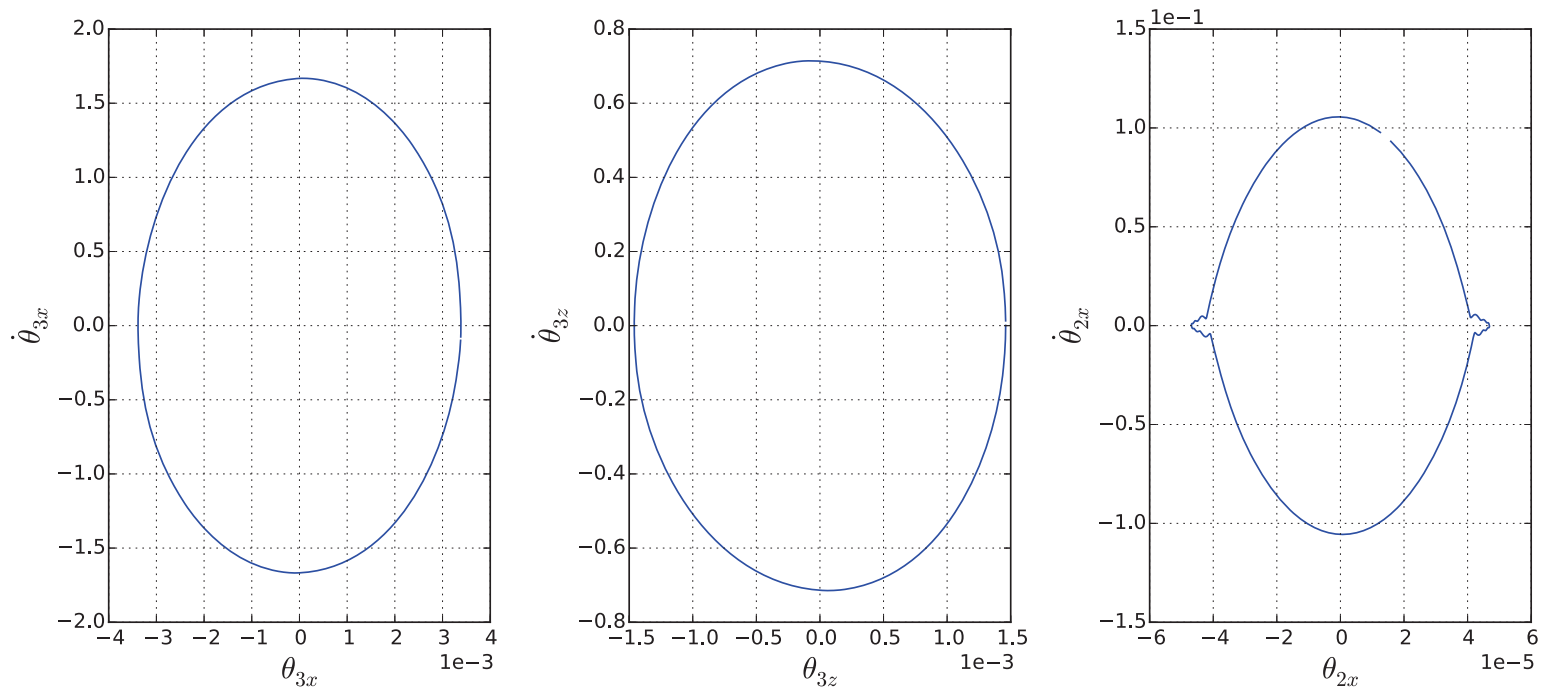

Figure 13: LCO computed by the means of nonlinear complex modes

\section{CONCLUSIONS}

This study has detailled a methodology to assess flutter stability of a fan stage while taking into account friction nonlinearities at blade root. An approach including the use of nonlinear complex modes and stability results from CFD calculations has been developped. It has been tested on a simple three degrees of freedom system that represents a blade-root-disk assembly. It allows to find a dry friction induced LCO while the system is aeroelastically unstable when no friction occurs. Moreover, the sensitivity of the aerodynamic damping to acoustic reflections in the inlet of the engine has been taken into account. The next step would be to apply this methodology in an industrial context, with a typical design of modern civil fan stage. However, some aspects that have been neglected in this study will have to be taken into account. Firstly, assumptions have been made for aeroelastic computations : the effects of the flow on the structure dynamics for the evaluation of aerodynamic forces due to vibration have been neglected. For structures as fan blades, which may be rather large and flexible for modern designs, these assumptions may not be totally realistic. Hence, it will be taken into account in a further study. Concerning the fluid modeling in the nonlinear complex mode computation, it has been chosen to add linear modal fluid effects only on the first harmonic of vibration. However, with increasing levels of energy, the nonlinear shape deformation may change sufficiently so that it has an impact on the flow. Hence, some data exchanges may have to be done between the nonlinear complex mode computation and the CFD based aeroelastic calculations to converge on each physics more accurately.

\section{ACKNOWLEDGEMENT}

The present study has been done thanks to the support of Safran Aircraft Engines. 


\section{REFERENCES}

[1] J.G. Marshall and M. Imregun, A Review of Aeroelasticity Methods with Emphasis on Turbomachinery Applications. Journal of Fluids and Structures, Vol. 10, No. 3, 237-267, 1996.

[2] H. Stargardter, Subsonic/transonic stall flutter study. NASA CR-165256, 1979.

[3] M. Vahdati et al. Mechanisms and prediction methods for fan blade stall flutter. Journal of Propulsion and Power, Vol. 17, No. 5, 1100-1108, 2001.

[4] R. Srivastava, M. A. Bakhle, T. G. Keith Jr. and G. L. Stefko, Flutter Analysis of a Transonic Fan. ASME Paper No. GT-2002-30319.

[5] M. Aotsuka and T. Murooka, Numerical analysis of fan transonic stall flutter. ASME Turbo Expo 2014: Turbine Technical Conference and Exposition, American Society of Mechanical Engineers (2014), Article V07BT35A020.

[6] X. Dong, Y. Zhang, Y. Zhang, Z. Zhang, X. Lu, Numerical simulations of flutter mechanism for high-speed wide-chord transonic fan. Aerospace Science and Technology, Vol. $105,2020$.

[7] S. W. Rienstra and A. Hirschberg, An introduction to acoustics. Eindhoven University of Technology, Vol. 18, 2004.

[8] M. Vahdati, G. Simpson and M. Imregun, Mechanisms for wide-chord fan blade flutter. Journal of Turbomachinery, American Society of Mechanical Engineers Digital Collection, Vol. 133, 2011.

[9] M. Vahdati, N. Smith and F. Zhao, Influence of intake on fan blade flutter. Journal of Turbomachinery, American Society of Mechanical Engineers Digital Collection, Vol. 137, 2015.

[10] F. Zhao, N. Smith and M. Vahdati, A simple model for identifying the flutter bite of fan blades. Journal of Turbomachinery, American Society of Mechanical Engineers Digital Collection, Vol. 139, 2017.

[11] C. Chahine, T. Verstraete, T and L. He, A comparative study of coupled and decoupled fan flutter prediction methods under variation of mass ratio and blade stiffness. Journal of Fluids and Structures, Vol. 85, 110-125, 2019.

[12] A. Sinha and J. Griffin, Friction Damping of Flutter in Gas Turbine Engine Airfoils. Journal of Aircrafts, Vol. 20, No. 4, 372-376, 1983.

[13] A. Sinha and J. Griffin, Effects of friction dampers on aerodynamically unstable rotor stages. AIAA journal, Vol. 23, No. 2, 262-270, 1985.

[14] R.M. Rosenberg, Normal modes of nonlinear dual-mode systems. 1960.

[15] R.M. Rosenberg, The normal modes of nonlinear n-degree-of-freedom systems. 1962.

[16] S.W. Shaw and C. Pierre, Normal modes for non-linear vibratory systems. Journal of sound and vibration, Vol. 164, No. 1, 85-124, 1993. 
[17] D. Laxalde and F. Thouverez, Complex non-linear modal analysis for mechanical systems: Application to turbomachinery bladings with friction interfaces. Journal of sound and vibration, Vol. 322, No. 4-5, 1009-1025, 2009.

[18] K. Malte, Nonlinear modal analysis of nonconservative systems: extension of the periodic motion concept. Computers \& Structures, Vol. 154, 59-71, 2015.

[19] E.P. Petrov, Analysis of flutter-induced limit cycle oscillations in gas-turbine structures with friction, gap, and other nonlinear contact interfaces. Journal of Turbomachinery, American Society of Mechanical Engineers Digital Collection, Vol. 134, No. 6, 2012.

[20] M. Lassalle and C.M. Firrone, A parametric study of limit cycle oscillation of a bladed disk caused by flutter and friction at the blade root joints. Journal of Fluids and Structures, Vol. 76, 349-366, 2018.

[21] C. Berthold, J. Gross, C. Frey and M. Krack, Analysis of Friction-Saturated Flutter Vibrations With a Fully Coupled Frequency Domain Method. Journal of Engineering for Gas Turbines and Power, Vol. 142, No. 11, 2020.

[22] L. Cambier, M. Gazaix, S. Heib, S. Plot, M. Poinot, J.P. Veuillot, J.F. Boussuge and M. Montagnac, An Overview of the Multi-Purpose elsA Flow Solver. AerospaceLab, 2011, p. 1-15. hal-01182452

[23] L. Cambier, S. Heib and S. Plot, The Onera elsA CFD software: input from research and feedback from industry. Mechanics \& Industry, Vol. 14, No. 3, 159-174, 2013.

[24] R. Henry, Calcul des fréquences et modes des structures répétitives circulaires. Journal de Mécanique Appliquée, Vol. 4, No. 1, 61-82, 1980.

[25] T. Bontemps, S. Aubert and N. De Cacqueray, Prediction of the acoustic influence of an intake on fan flutter: a comparison of numerical methods. 13th European Conference on Turbomachinery Fluid dynamics \& Thermodynamics, European Turbomachinery Society, 2019.

[26] A. H. Nayfeh and B. Balachandran, Applied nonlinear dynamics: analytical, computational, and experimental methods. John Wiley \& Sons, 2008.

[27] T.M. Cameron and J.H. Griffin, An alternating frequency/time domain method for calculating the steady-state response of nonlinear dynamic systems. American Society of Mechanical Engineers, Journal of Applied Mechanics, Vol. 56, No. 1, 149-154, 2019.

[28] K.B. Blair, C.M. Krousgrill and T.N. Farris, Harmonic balance and continuation techniques in the dynamic analysis of Duffing's equation. Journal of Sound and Vibration, Vol. 202, No. 5, 717-731, 1997. 